$7^{\text {th }}$ Jagna International Workshop (2014)

International Journal of Modern Physics: Conference Series

Vol. 36 (2015) 1560016 (7 pages)

(C) The Authors

DOI: $10.1142 /$ S2010194515600162

\title{
Fractional Brownian motion and polymers: Learning from each other
}

\author{
J. Bornales \\ Physics Department, Mindanao State University-Iligan Institute of Technology, \\ Iligan City, 9200 Philippines \\ jinky.bornales@g.msuiit.edu.ph \\ L. Streit \\ Forschungszentrum BiBoS, Bielefeld University, D 33501 Bielefeld, Germany, \\ and \\ CCM, University of Madeira, P 9000-390 Funchal, Portugal \\ streit@physik.uni-bielefeld.de
}

Published 2 January 2015

\begin{abstract}
Self-avoiding or self-repelling random paths, with motivation from their use in polymer physics, have been widely studied using the tools of mathematics, physics, and computer simulations. We illustrate this by three recent examples.
\end{abstract}

\section{Introduction}

Self-avoiding or self-repelling random path models for polymer configurations have been studied extensively in mathematics using combinatorics, stochastic analysis, in statistical mechanics, and in computer physics using Monte Carlo methods. Classical texts are e.g. ${ }^{4}$ and ${ }^{12}$, a recent review can be found $\mathrm{in}^{17}$. The field is highly interdisciplinary: the motivation came from chemistry, while physics provides structural intuition and far-reaching predictions, and computer simulations can check them out. The mathematical results are less far-reaching but provide the higher reliability characteristic of the mathematical approach; much remains to be done in the stochastic analysis setting.

Apart from self-avoiding random walks, a prominent realization is the Edwards model of self-repelling (or "weakly self-avoiding") Brownian paths, an example of models where self-crossings are not strictly forbidden but where there is an exponential penalty on self-crossings. ${ }^{8}$

This is an Open Access article published by World Scientific Publishing Company. It is distributed under the terms of the Creative Commons Attribution 3.0 (CC-BY) License. Further distribution of this work is permitted, provided the original work is properly cited. 
Recently the Edwards model has been extended to fractional Brownian motion $(\mathrm{fBm}),{ }^{13}$ allowing for models of stiffer or curlier polymers than those described by classical Brownian motion.

It is in this context that we shall present report on progress on three fronts, where methods from stochastic analysis, arguments from statistical physics, and numerical computation have been employed.

\section{Recent developments}

\subsection{Varadhan's existence proof}

In the Edwards model self-repelling paths are described via a "Boltzmann factor" to suppress self-intersections of Brownian motion $B$ :

$$
G=\frac{1}{Z} \exp \left(-g \int_{0}^{N} d s \int_{0}^{N} d t \delta(B(s)-B(t))\right)
$$

with

$$
Z=E\left(\exp \left(-g \int_{0}^{N} d s \int_{0}^{N} d t \delta(B(s)-B(t))\right)\right)
$$

The mathematical problem here lies with the existence of this exponential, how to make sense of

$$
L=\int_{0}^{N} d s \int_{0}^{N} d t \delta(B(s)-B(t))
$$

One uses delta sequences to approximate the Dirac distribution

$$
\begin{aligned}
& \delta_{\varepsilon}(x):=\frac{1}{(2 \pi \varepsilon)^{d / 2}} e^{-\frac{|x|^{2}}{2 \varepsilon}}, \quad \varepsilon>0, \\
& L_{\varepsilon}:=\int_{0}^{l} d t \int_{0}^{t} d s \delta_{\varepsilon}(B(t)-B(s)) .
\end{aligned}
$$

Removing the regularization depends on the dimension of the Brownian paths, it is straightforward for $d=1$ but for $d \geq 2$ the expectation diverges

$$
\lim _{\varepsilon \searrow 0} \mathbb{E}\left(L_{\varepsilon}\right)=\infty
$$

In $d=2$ one needs to subtract the expectation, setting

$$
L_{\varepsilon}^{c}=L_{\varepsilon}-\mathbb{E}\left(L_{\varepsilon}\right)
$$

and will define the centered self-intersection local time as

$$
L^{c}=\lim _{\varepsilon \searrow 0} L_{\varepsilon}^{c}
$$

In $d=3$ a further, multiplicative renormalization is required, ${ }^{20}$ but for $d=2$ one is thus led to consider

$$
\exp \left(-g L^{c}\right)
$$


However, after centering $L^{c}$ is unbounded below, and

$$
\exp \left(-g L^{c}\right) \rightarrow \infty \text { when } L^{c} \rightarrow-\infty \text {. }
$$

So one needs to show that large values occur only with small probability such that the expectation is nevertheless finite. Varadhan ${ }^{19}$ shows that

$$
\mathbb{E}\left(\exp \left(-g L^{c}\right)\right)<\infty
$$

and a bona fide probability distribution for planar self-repelling Brownian paths exists if $g>0$ is sufficiently small. By a further scaling argument this existence result can be extended to all $g>0$.

Varadhan's famous proof is based on a clever use of the Chebyshev inequality, using the logarithmic divergence of the expectation and an estimate of the rate of convergence

$$
\left\|L^{c}-L_{\varepsilon}^{c}\right\|_{L^{2}(\mu)}^{2} \leq \text { const. } \varepsilon^{a} \text { for all } a<1 / 2 .
$$

Concerning this rate of convergence, in his words, "...this is the most difficult step of all and requires considerable estimation". Hence an alternate proof may be of interest.

Using the tools of White Noise Analysis, in the particular multiple Wiener integral or "chaos" decomposition of the self-intersection local time ${ }^{9}$ it is straightforward to show ${ }^{2}$

Theorem 2.1. Let $T>0$ be given. Then

$$
\left\|L_{\varepsilon}^{c}(T)-L^{c}(T)\right\|^{2} \leq C \varepsilon^{\alpha} \forall \alpha<1 .
$$

Apart from a simplified proof one notes the doubling of the convergence rate $\alpha$.

Remark 2.1. While a generalisation of the Varadhan existence proof to $\mathrm{fBm}$ is now available, ${ }^{13}$ its extension to arbitrary coupling constants $g$ remains an open challenge.

\subsection{The Flory index}

Contrary to the above, the scaling behavior of self-repelling paths is not (yet) accessible to strict mathematical arguments, with the exception of the unphysical one-dimensional case. ${ }^{14}$

The question, in physical terms, is about the "end-to-end-length" $\mathrm{R}$ of polymer, and how it grows as one increases the number $N$ of monomers in the chain. Mathematically, for a (fractional) Brownian path $x=B^{H}$, one has

$$
\mathbb{E}\left(x(N)-x(0)^{2}\right)=N^{2 \nu}
$$

with $\nu$ equal to the "Hurst index" $H$ which characterizes the fBm. The suppression of self-intersections, "excluded volume effect" in physics terminology, will make the paths swell and one expects $\nu>H$. 
Flory's famous formula ${ }^{10}$

$$
\nu=\frac{3}{d+2}
$$

for spatial dimensions $d=1,2,3$ was based on a flawed mean field argument ${ }^{12}$ but is remarkably accurate. ${ }^{17}$ Recently it was extended to the fractional case, ${ }^{1}$ with

$$
\nu_{H}=\frac{2 H+2}{d+2} .
$$

Computer simulations appear to support this formula at least for $d=1$. In Refs. ${ }^{5}$ and $^{6}$ the original self-avoiding Brownian model has been extended to the case where $k$-fold intersections are tolerated, and only higher order ones penalized. Informally this would correspond to the use of higher order self-intersection local times

$$
L^{(k)}(N) \equiv \int_{0}^{N} d t \int_{0}^{N} d^{k} s \prod_{l=1}^{k} \delta\left(x(t)-x\left(s_{l}\right)\right) .
$$

For a rigorous mathematical discussion of such higher order intersection local times and the necessary renormalizations to make them well-defined see e.g. ${ }^{7}$.

Putting aside the problems of a rigorous definition, one can invoke, simple, dimensional arguments would lead to

$$
\nu_{H, k}(d)=\frac{2 H+k+1}{k d+2}
$$

which now covers all values of the dimension $d$, the Hurst index $H$, and the tolerance level $k$, up to the limiting dimensions where there are no more self-intersections, see formula (3) below.

The singularity of higher order self-intersection local times and the nonMarkovian nature of $\mathrm{fBm}$ complicate the mathematical analysis of scaling even beyond the classical Brownian case. In this light it is remarkable that for

$$
H d=\frac{k+1}{k}
$$

the formula (2) produces

$$
\nu_{H, k}(d)=H
$$

i.e. there is no swelling from the suppression of self-intersections. This coincides in fact with a rigorous mathematical result: Talagrand ${ }^{18}$ proves that indeed with probability $1 \mathrm{fBm}$ has no $(k+1)$-tuple points whenever

$$
H d \geq \frac{k+1}{k} .
$$

On the other hand one notes that for $d=1$ the formula (2) would predict increased swelling for $k>1$; also in the Bm case one expects Wilson type renormalization to induce a $k=1$ term in the renormalized interaction, ${ }^{16}$ hence the usual Flory scaling also for $\mathrm{k}$-tolerant models. 


\subsection{Computational results}

In view of the difficulties confronting a mathematical investigation computer simulations suggest themselves.

Results in this direction are based on a discretized version of the k-tolerant model, with monomer positions

$$
x_{k}=B^{H}(k), k=0,1,2, \ldots, N-1 .
$$

As for the self-intersection local time $L^{(k)}$, one notes that, informally, we can express it in terms of the local time $L(N, u)$, given informally by

$$
L(N, u)=\int_{0}^{N} d t \delta(x(t)-u)
$$

Indeed one has

$$
\begin{aligned}
L^{(k)}(N) & =\int_{0}^{N} d t \int_{0}^{N} d^{k} s \prod_{l=1}^{k} \delta\left(x(t)-x\left(s_{l}\right)\right) \\
& =\int_{0}^{N} d u\left(\int_{0}^{N} d t \delta(x(t)-u)\right)^{k+1} \\
& =\int_{0}^{N} d u L^{k+1}(N, u)
\end{aligned}
$$

and the latter is straightforward to discretize. Monte Carlo computations with importance sampling ${ }^{15}$ are then based on a conformation energy

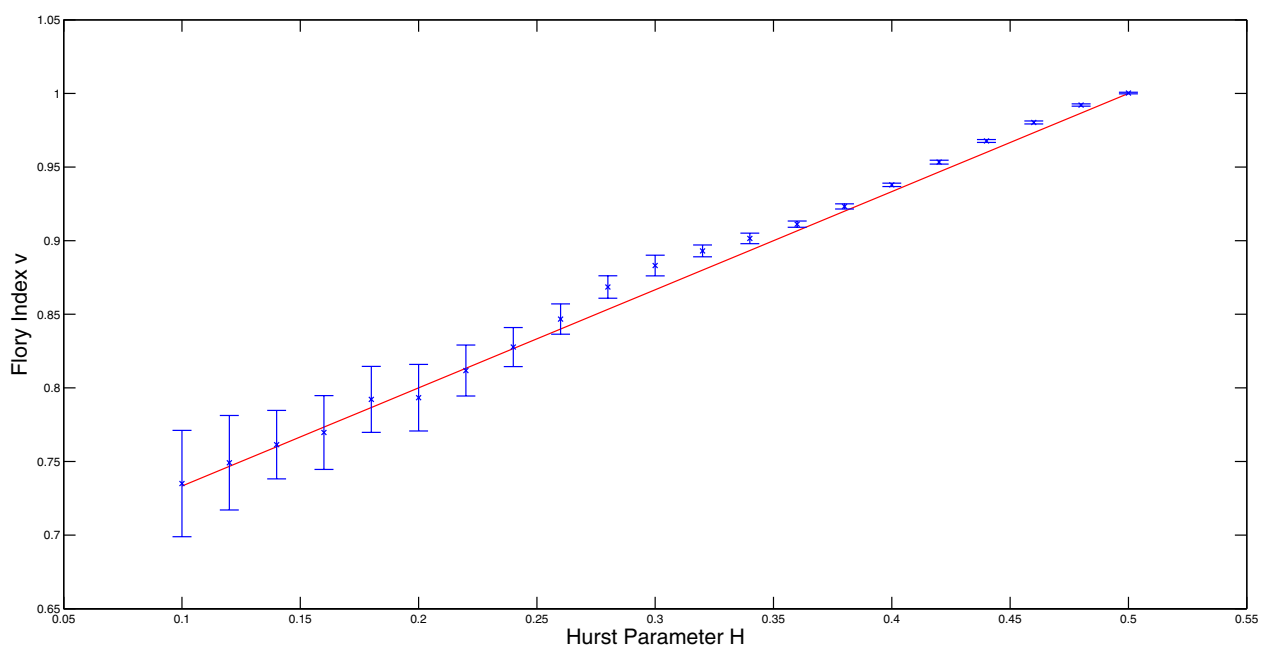

Fig. 1. 


$$
E(x)=E_{0}(x)+g E_{1}(x)
$$

where $E_{0}$ is obtained by inverting the fBm covariance matrix, and the excluded volume energy is given by a discretized version of $L^{(k)}$.

Preliminary results for $d=1, k=1$, and $0<H<1 / 2$ give encouraging results, see Fig. 1. The straight line corresponds to formula (2) for $k=1$; extensive precision calculations are under way ${ }^{3}$.

\section{Acknowledgments}

This work was financed by Portuguese national funds through FCT - Fundação para a Ciência e Tecnologia within the project PTDC/MAT-STA/1284/2012.

\section{References}

1. J. Bornales, M.J. Oliveira, L. Streit: Self-repelling fractional Brownian motion - a generalized Edwards model for chain polymers. In L. Accardi, W. Freudenberg, M. Ohya (Eds.), Q. Probability and WNA 30, 389-401. World Scientific Singapore, 2013.

2. W. Bock, M.J. Oliveira, J.L. Silva, L.Streit: Polymer Measure: Varadhan's Renormalization Revisited, preprint, 2014. arXiv:1405.7150 [math-ph]

3. W. Bock, J. Bornales, S. Eleuterio (to be published).

4. J. des Cloiseaux and G. Jannik, Polymers in Solutions: Their Modelling and Structure. Oxford University Press 1990.

5. R. Dekeyzer, A. Maritan, A. Stella, Excluded-volume effects in linear polymers: Universality of generalized self-avoiding walks. Phys. Rev. B 31, 4659-4662 (1985).

6. R. Dekeyzer, A. Maritan, A. Stella, Random walks with intersections: Static and dynamic fractal properties. Phys. Rev. A 36, 2338-2351 (1987).

7. S. Mendonça, L. Streit: Multiple Intersection Local Times in Terms of White Noise. IDAQP 4, 533 (2001).

8. S. F. Edwards, The statistical mechanics of polymers with excluded volume. Proc. Roy. Soc. 85, 613-624 (1965).

9. M. Faria, T. Hida, L. Streit, H. Watanabe: Intersection Local Times as Generalized White Noise Functional. Acta Appl. Math. 46, 351 (1997).

10. P.J. Flory, Principles of Polymer Chemistry. Cornell University Press. 1953

11. M.E. Fisher, J. Phys. Soc. Japan 26 Suppl. 44 (1969)

12. P. G. de Gennes, Scaling Concepts in Polymer Physics. Cornell Univ. Press, Ithaca, NY. (1979)

13. M. Grothaus, M.J. Oliveira, J.-L. Silva, L. Streit: Self-avoiding fBm - The Edwards model. J. Stat. Phys. 145, 1513-1523 (2011).

14. R. van der Hofstad, W. König, A Survey of One-Dimensional Random Polymers. J. Stat. Physics, 103, 915-944 (2001).

15. N. Metropolis, A.W. Rosenbluth, M.N. Rosenbluth, A.H. Teller, and E. Teller: Equation of State Calculations by Fast Computing Machines. Journal of Chemical Physics 21, 1087-1092 (1953).

16. Y. Oono, K. Freed: Conformation Space Renormalizationof Polymers I. J. Chem. Phys. 75, 993-1008 (1981).

17. A. Pelissetto, E. Vicari: Critical phenomena and renormalization-group theory. Phys. Reports 368, 549-727 (2002).

18. M. Talagrand: Multiple points of trajectories of multiparameter fractional Brownian motion. Probab. Theory Related Fields 112, 545-563 (1998). 
19. S. R. S. Varadhan: Appendix to "Euclidean quantum field theory" by K. Symanzik, in: R. Jost, ed., Local Quantum Theory, Academic Press, New York, p. 285 (1970)

20. M. J. Westwater: On Edwards' model for long polymer chains. Comm. Math. Phys.72, 103-205 (1980). 